\title{
SUPPRESSION OF COMMON-MODE RADIATION FROM HIGH SPEED PRINTED CIRCUIT BOARD USING MODIFIED GROUND PATTERNS
}

\author{
Dr.N.Suresh kumar ${ }^{1}$, V.Anandha jothi ${ }^{2}$, Darshini.B ${ }^{2}$, S.Raja Suhanya ${ }^{2}$, \\ P.Rajeswari ${ }^{3}$ \\ ${ }^{1}$ Principal ,Velammal College of Engineering and Technology Madurai-625009,India. \\ ${ }^{2}$ UG Students,ECE Department, Velammal College of Engineering And \\ Technology,Madurai-625009,India. \\ ${ }^{3}$ Assistant Professor,ECE department,Velammal College of Engineering and \\ Technology,Madurai-625009,India
}

\begin{abstract}
-
Now-a-days, the usage for the high speed printed circuit boards are increased. Inorder to satisfy the high demand of low cost and size reduction, chips must be integrated in a same package in which the signal integrity issues may occur. One of the major issues is radiated emission. The common mode and differential mode radiation occur when the differential signal crosses the splitted reference plane. In this paper, to reduce the common mode radiation, asymmetric resonators with the interdigital capacitors on the splitted ground plane with shorting vias is implemented. The physical dimensions for the stub and the interdigital capacitors are determined by using the design theory of transmission line model. The insertion loss, return loss and the field pattern are determined using the Ansoft HFSS Tool.
\end{abstract}

\section{Keywords-}

Common mode radiation, Electromagnetic Interference (EMI), Interdigital Capacitors (IDC), Radiated Emission, Split plane.

\section{INTRODUCTION}

In the high speed printed circuit board, the chips must be integrated in a single package. Electronic devices are becoming light weight and so demands of miniaturizing PCBs in this device increase. Now-a-days signal integrity has become a major issue. The signal quality is more important for the high speed products and the signal integrity analysis should ensure reliable transmission. The signal integrity issues include ringing, crosstalk, ground bounce, radiated emission and reflection [1]. One of the major issues is radiated emission. The radiated emission is the unintentional release of electromagnetic energy from a device. The emission from one device may interfere with the neighbouring device which would cause the circuit malfunction. The main source of the radiated emission is the common mode current. This radiated emission can be reduced by using the stitching capacitors [5]. It provides return current path of signal lines. It degrades the noise isolation or they suppress the signal distortion and decreases radiation 
International Journal of Information Sciences and Techniques (IJIST) Vol.4, No.3, May 2014

emission. But it may cause internal reflection and resonance. By decreasing the distance between the ground plane and the signal traces, the radiation can be reduced but it may induce the coupling mechanism. Inorder to decrease the coupling, the ground guard fence was used between the microstrip lines and the disadvantage in using the ground guard fence is that, it increases the size of the printed circuit board. It is common to introduce slots in the reference plane to provide the DC isolation [6]. An asymmetrical grounded resonators technique was proposed to reduce the EMI for slot crossing differential signals. These asymmetric resonators with one end shorting to the ground plane provides efficient shorting path between the split planes at different frequencies. The vias were used at the one end of the resonators to electrically connect the resonators and the ground plane [1].

In this paper, the asymmetric grounded resonators with interdigital capacitors is proposed to decrease the slot crossing differential signals. The grounded resonators are placed on the PCB by designing a stub with a shorting via connecting to the ground. The interdigital capacitors are placed at one end of the stub. These resonators provide the return current path and would acts as an inductor. The interdigial capacitors provide capacitance and this capacitance and inductance get cancelled and thus reduces the radiated emission.

\section{RADIATED EMISSION ON PCB}

Radiated emission is one of the major issues of signal integrity. Radiated emission is the unintensional discharge of electromagnetic energy from an electronic device. Radiated emission occurs when there is a via or a split or a bend in a trace. Emission from one device may cause malfunction in the neighbouring device. The main source of radiated emission is common mode noise. Common mode radiation and differential mode radiation arises when a differential signal crosses a slotted reference plane. The differential mode current and common mode current occurs in a parallel conductor. Common mode current are equal in magnitude and same in direction where as the differential mode current are equal in magnitude and opposite in direction. Common mode noise arises when the signal trace radiates energy with respect to the ground. It is result of undesired voltage drop in the circuit that causes some parts of the system to be at a common mode potential and it is harder to control than the differential mode radiation. Differential mode radiation is the result of current flowing around the loop formed by the conductor of the circuit. The common mode radiation can be reduced by minimizing the common mode current. This common mode current is controlled by minimizing the source voltage that drives the antenna, providing large common mode choke in series with the cable, shunting the current to ground and by shielding the cable. Fig 1 and Fig 2 shows the existing method. The insertion loss is about $8 \mathrm{~dB}$ and the return loss is $-10 \mathrm{~dB}$. They are measured at a frequency of $4 \mathrm{GHz}$.

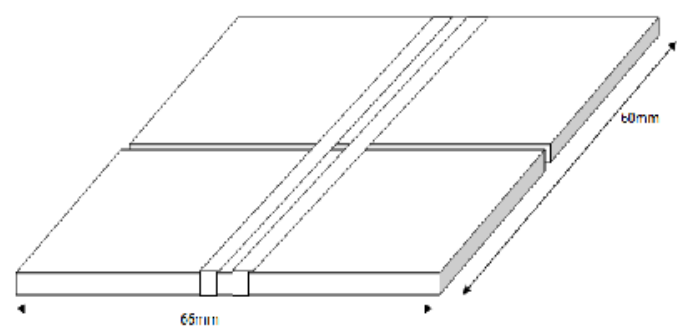

Fig. 1. Coupled microstrip in a splitted reference plane 
Fig 1 shows the coupled microstrip placed on the FR4 epoxy substrate near the differential lines on a splitted reference plane.

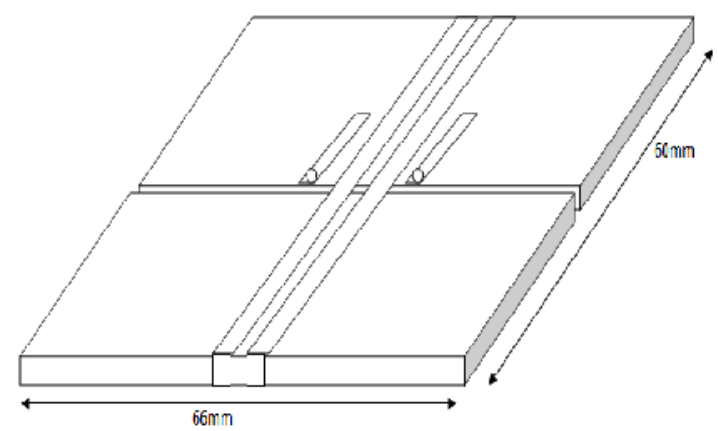

Fig 2. Asymmetric grounded resonators in a splitted reference plane with stub

Fig 2 shows that the vias are placed at one end of the asymmetric grounded resonators which are placed on the FR4 epoxy substrate near the differential lines on a splitted reference plane. In this method the return loss is $-8.3 \mathrm{~dB}$ and the insertion loss is about $-12 \mathrm{~dB}$.

\section{PROPOSED METHOD}

The asymmetrical grounded resonators with interdigital capacitors and a shorting via are placed in a splitted reference plane. The method of realising a capacitor is by providing a slot in the middle of the conductive strip. This will act as a parallel plate capacitor which is known as the interdigital capacitor (IDC).

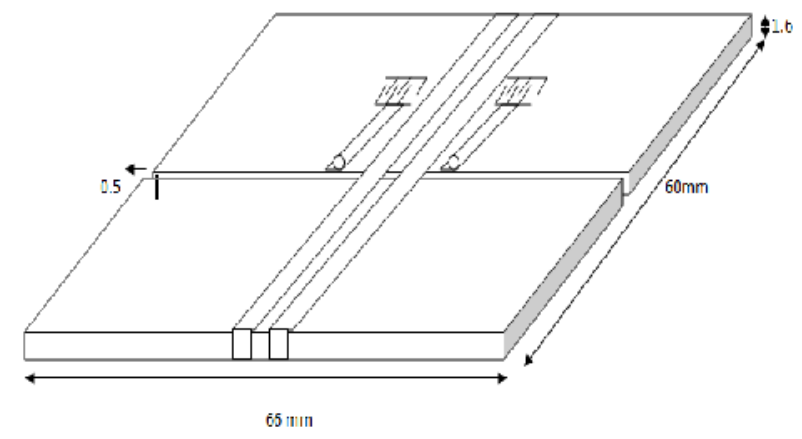

Fig 3Asymmetric grounded resonators in a splitted reference place with IDC

The physical structure is shown in the Fig 3. The differential line with width, W and a space, S aymmetrically crosses its split reference plane with a width of, Ws. The distance between the centre of the differential line and the both end of the split is given by Lo and Ls respectively. The length and width is given by $L_{g n d}$ and $W_{g n d}$. The height of substrate is $\mathrm{H}$. The relative permittivity of the dielectric is $\varepsilon_{r}$. The grounded resonators are kept in parallel to the differential line in which one end of them is shorted with vias and the other end is connected with the interdigital capacitors. The width of the resonators is $W_{g r}$. The distance from the centre of the 
spacing between the differential lines to the resonator is given by $S_{g r}$ and the dissimilar length of the resonators are denoted by $L_{\text {gro }}$ and $L_{\text {grs }}$. These two grounded resonators have same characteristic impedance $Z_{g r}$ and a phase constant of $\beta_{g r}$ used to control the transmission frequency. The resonators are designed using the following equations [1]. The characteristic impedance $Z_{O S}$ is given as,

$$
\begin{aligned}
& Z_{o s}=\quad 73.6-2.15 \varepsilon_{r}+\left(638.9-1.37 \varepsilon_{r}\right)\left(\frac{W S}{\lambda o}\right)^{0.6}+\left(36.23 \sqrt{\left.\left(\varepsilon^{2}{ }_{r}+41\right)-225\right)} \frac{W S / H}{\left(\frac{W S}{H}+0.876 . \varepsilon_{r}-2\right)}\right. \\
& +0.51\left(\varepsilon_{r}+2.12\right)\left(\frac{W S}{H}\right) \ln \left(100 \frac{H}{\lambda o}\right)-0.753 \varepsilon_{r} \frac{\left(\frac{H}{\lambda o}\right)}{\sqrt{W S} / \lambda o}
\end{aligned}
$$

And the phase constant,

$$
\beta s=2 \pi / \lambda s
$$

Where $\lambda o$ is defined as

$$
\begin{aligned}
& \frac{\lambda s}{\lambda o}=0.9217-0.277 \ln \left(\varepsilon_{r}\right)+0.0322\left(\frac{W S}{H}\right)\left(\frac{\varepsilon_{r}}{\frac{W S}{H}+0.435}\right) \wedge 0.5-0.01 \ln \left(\frac{H}{\lambda o}\right)[4.6- \\
& \left.\frac{3.65}{\varepsilon_{r}^{2} \sqrt{W S} / \lambda o\left(9.06-100 \frac{W S}{\lambda o}\right)}\right]
\end{aligned}
$$

$\lambda o$ is the wavelength of vacuum.

The gap between the interdigital capacitors are equal and if the gap between it decreases then the capacitance increases. Reducing the width of the finger reduces the required area but decreases the characteristic impedance of the line and so the effective capacitance decreases. If the length of the finger increases then the capacitance increases and so the board area gets increased. The number of fingers may range from 2 to 200 .

The design of the interdigital capacitors is described. The effective capacitance can be found by three capacitive structures as shown in Fig 4.

$$
\begin{gathered}
C_{1}=\varepsilon_{0} \varepsilon_{e f f} * \frac{K\left(K_{01}^{\prime}\right)}{K\left(K_{01}\right)} * \mid \\
K_{01=\sqrt{1-\left(\frac{g}{g+s}\right)} 2}
\end{gathered}
$$




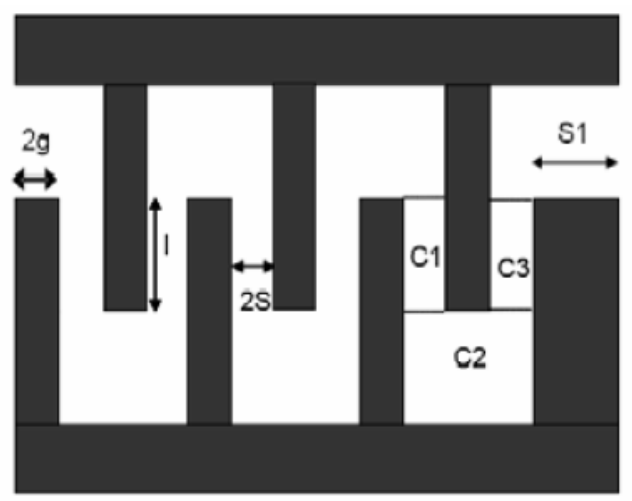

Fig 4 . Interdigital Capacitors

$C_{2}=2 \varepsilon_{0} \varepsilon_{\text {eff }} * \frac{K\left(k_{01}\right)}{K\left(K_{01}^{\prime}\right)} * l_{\text {ext }}$

Where,

$l_{\text {ext }}=1 / 4$

$c_{3}=4 \varepsilon_{0} \varepsilon_{e f f} * \frac{k\left(k_{02}^{\prime}\right)}{k\left(k_{02}\right)} * 1$

$k_{02}=\sqrt{\frac{s 1 * s}{(2 g+s) *(2 g+s 1)}}$

$\mathrm{C}=(\mathrm{n}-3) c_{1}+(\mathrm{n}-1) c_{2}+2 c_{3}$

$\varepsilon_{e f f}=1+\frac{\varepsilon_{r-1}}{2}$

\section{RESULTS AND DISCUSSIONS}

The ground plane has a dimension of $60 \mathrm{x} 66 \mathrm{~mm}$. The height of the FR4 epoxy substrate is about $1.6 \mathrm{~mm}$. The length and width of the differential line is about $60 \mathrm{~mm}$ and $1.27 \mathrm{~mm}$. The width of the split is given by $0.5 \mathrm{~mm}$. The asymmetric grounded resonators L_gro and L_grs are said to be $9.3 \mathrm{~mm}$ and $12.5 \mathrm{~mm}$. The distance between the two differential lines is $0.51 \mathrm{~mm}$. The $\mathrm{S}_{-}$(gr )is about $10 \mathrm{~mm}$. The scattering parameters are used to study the characteristics of common mode radiation. The return loss and insertion loss are defined by $S_{11}$ and $S_{21}$. They are derived by using the following equations [4].

$S_{c c 11}(\mathrm{~dB})=20 \log \left[\frac{1}{2}\left(S_{11}+S_{21}+S_{12}+S_{22}\right) \mid\right.$
$S_{c c 21}(\mathrm{~dB})=20 \log \left[\frac{1}{2}\left(S_{31}+S_{41}+S_{32}+S_{42}\right) \mid\right.$ 
The resonators which are used are inductive in nature. The interdigital capacitors placed above the resonators have a capacitve effect and thus cancells with each other.

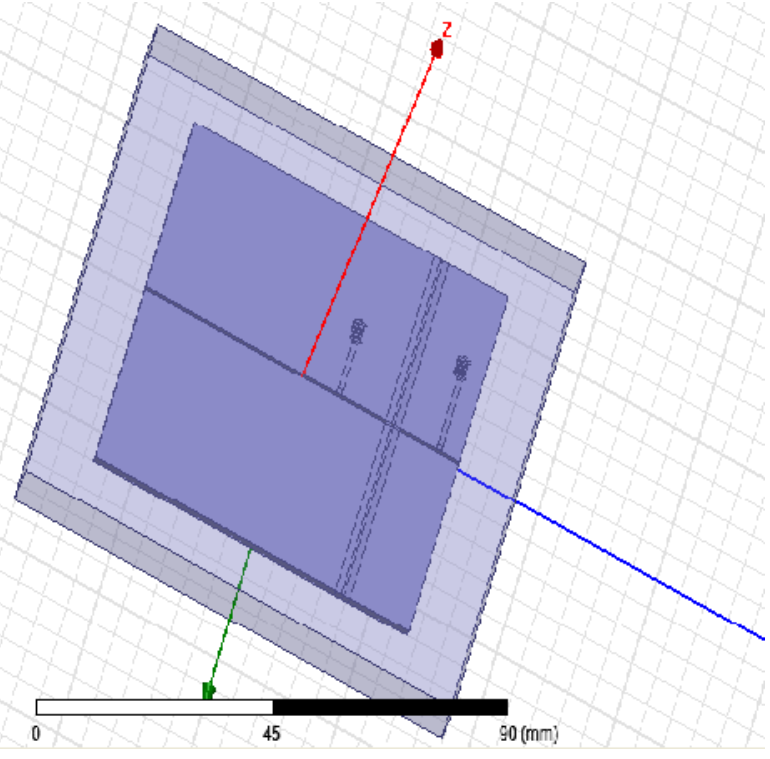

Fig 5. Proposed structure in Ansoft HFSS tool

The proposed structure is implemented using the Ansoft HFSS tool snapshot is shown in the Fig 5. The Fig 6 and Fig 7 show the return loss and inserion loss of the proposed and the conventional models. These scattering parameters are simulated for the frequency range of 0 to $4 \mathrm{GHz}$. For the proposed model the insertion loss is $-14 \mathrm{~dB}$ and the return loss is found to be $-2 \mathrm{~dB}$.

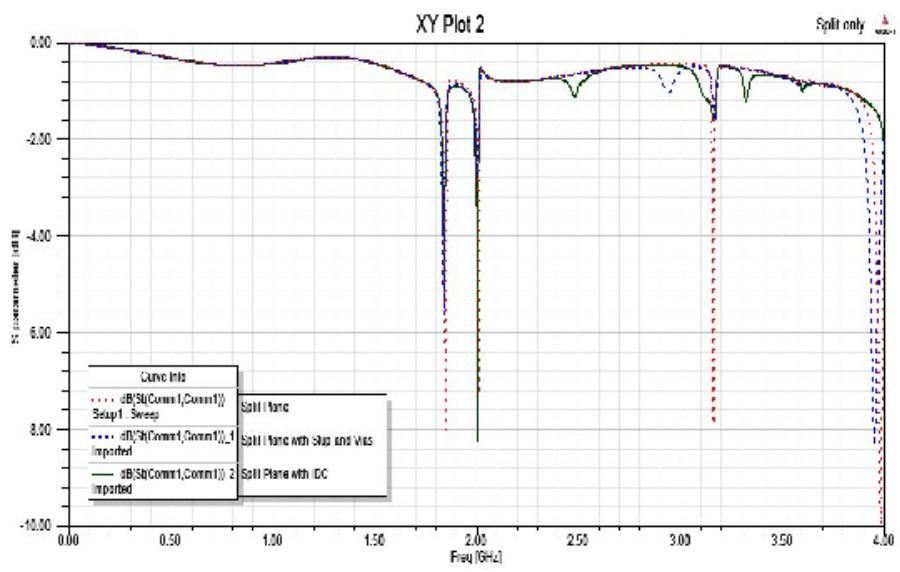

Fig 6. Compared results of the return loss for the splitted reference plane, splitted plane with a vais and the splitted reference plane with IDC 


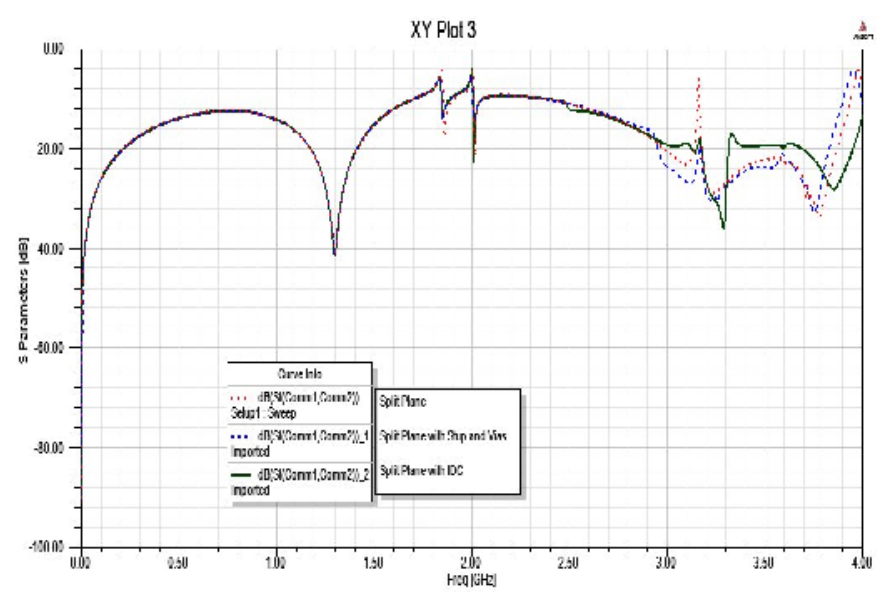

Fig 7. Compared results of the insertion loss for the splitted reference plane, splitted plane with a vias and the splitted reference plane with IDC

\section{CONCLUSION}

Thus the asymmetrical grounded resonators with the interdigital capacitors is designed and proposed using the Ansoft HFSS tool. The return loss, insertion loss and the field pattern are simulated for the proposed structure. At $4 \mathrm{GHz}$, the return loss shows a reduction of about $6 \mathrm{~dB}$ and the insertion loss increases approximately $2 \mathrm{~dB}$. The physical dimensions for the stub and the interdigital capacitors are determined by using the design theory.

\section{REFERENCES}

[1] Hao-Hsiang Chuang and Tzong-Lin Wu, Senior member IEEE, "Suppression of common- mode radiation and mode conversion for slot crossing $\mathrm{GHz}$ Differential signals Using Novel grounded Resonators" ,IEEE Transactions on Electromagnetic compatability, Vol.53,No.2,May 2011.

[2] A.K. Arya, A.Patnaik, M.V.Karthikeyen, "A compact array with low mutual coupling using defected ground structures" in IEEE Conference, 2011.

[3] Hao-Hsiang Chuang and Tzong-Lin Wu, Senior member IEEE, "A Novel ground resonator technique to reduce common mode radiation on slot-crossing differential signals" in IEEE Microwave and wireless Components letters,Vol.20,No.12,December 2010.

[4] Hao-Hsiang Chuang and Tzong-Lin Wu, Senior member IEEE, "A new common mode EMI Suppression technique for $\mathrm{GHz}$ differential signals crossing slotted reference planes" in IEEE conference, 2010.

[5] Jingook Kim, Student Member, IEEE, Heeseok Lee and Joungho Kim, Associate member, IEEE,"Effects on Signal Integrity and Radiated Emission by Split Reference Plane on High- Speed Multilayer Printed Circuit Boards", in IEEE Transactions on Advanced Packaging,Vol.28,No.4,November 2005 .

[6] Hitoshi Nagakubo,Kimitoshi Murano,Fengchao Xiao, Yoshio Kami, "Circuit Model for Two Parallel Microstrip Lines on Slotted Ground Plane”, in IEEE Conference in 2007.

[7] Guang-Hwa Shiue, Member, IEEE, and Ruey-Beei Wu, Senior Member, IEEE,'Reduction in Reflections and Ground Bounce for Signal line Over Slotted Power Plane Using Differential coupled Microstrip Lines" in IEEE Transactions on Advanced Packaging,Vol.32,No.3,August 2009.

[8] Mark L Montrose, Jin Hong-Fang, Er-Ping Li,"Analysis on the Effectiveness of High Speed Printed Circuit Board Edge Radiated Emissions Based on Stimulus Source Location” in IEEE Conference.

[9] Sergiu Radu and David Hockanson,"An Investigation of PCB Radiated Emissions from Simultaneous Switching Noise" in IEEE Conference, 1999. 
[10] Mark I. Montrose, "Analysis on Loop Area Trace Radiated Emissions from Decoupling Capacitor Placement on Printed Circuit Boards", in IEEE Conference, 1999.

[11] Junho Lee, Hyungsoo Kim, and Joungho Kim, "Broadband Suppression of SSN and Radiated Emissions using High-DK Thin Film EBG Power Distribution Network for High-Speed Digital PCB Applications", in IEEE Conference, 2005.

[12] Changyi Su, Student Member, IEEE, and Todd H. Hubing,"Calculating Radiated Emissions Due to I/O Line Coupling on Printed Circuit Boards Using the Imbalance Difference Method".

[13] Bert Wong, Antonio Cantoni,"Radiated Emissions and Signal Integrity of Printed Circuit Board Microstrips", in IEEE Conference. 